\title{
A Reassessment of the Role of Membranes in the TMV Replication Complex
}

\author{
R. Howard Berg, Maria Soto-Aguilar, and Roger N. Beachy \\ Donald Danforth Plant Science Center, 975 North Warson Road, St. Louis, MO, 63132
}

We are interested in understanding how viruses harness plant cell function to reproduce, using tobacco mosaic virus (TMV) as a model. TMV replication occurs in viral replication complexes (VRCs), assembled in association with the ER [1]. TMV gene products include replicase (126 $\mathrm{kDa}$ and $183 \mathrm{kDa}$ proteins, Rep) and movement protein (30 kDa, MP). GFP localization and immunofluorescence show these proteins occupy distinct cellular locations initially, eventually colocalizing in bodies up to several micrometers in diameter (Fig. A). These bodies colocalize with ER 14-18 hours after infection, in VRCS surrounding the nucleus. VRCs are dynamic, moving on actin cables (Fig. B). Eventually moving to the cell periphery, VRCs associate with plasmodesmata, which are the avenues for cell to cell spread of the virus $[2,3]$.

Domains of MP required for its association with membranes have been identified [4]. The association of MP with ER in VRCs, and the presence of a putative transmembrane domain in MP, has led to conjecture that MP is an integral ER membrane protein [3].

To provide a stronger foundation for modeling VRC function, we have used electron microscopy to examine the ultrastructure of VRCs in thin sections and also using electron tomography, in tobacco leaves infected with TMV. Samples were fixed by high pressure freezing and embedded in resin after freeze substitution. Corroborating data from light microscopy, membranes within VRCs are in the form of (rough) ER cisternae, which are scattered throughout VRCs (Figs. C,D). There is a high density of free ribosomes in VRCs. Viral proteins form complexes, "ropes" (Figs. C,D), that ramify within the VRC and contain Rep (Fig. E) and perhaps MP [5]. These have been observed in chemically fixed tissue as well [6]. Electron tomography made it possible to model in 3D the relationship of viral ropes with the ER (Figs. F,G). Based on this information, there is little evidence that the bulk of viral protein (ropes), including MP, is directly associated with the ER membrane. Instead, the ropes are associated with free and ER-bound ribosomes, via linker bridges (Figs. F,G). This calls for a reevaluation of how MP associates with VRC membranes. Ongoing work is aimed at thick section immunogold tomography, to identify whether the linker bridges contain MP or Rep, and to map the distribution of these two proteins in the VRC complex.

[1] P. Mas and R.N. Beachy, J Cell Biol 147 (1999) 945.

[2] S. Kawakami et al., PNAS 101 (2004) 6291.

[3] D. Guenoune-Gelbart et al., MPMI 21 (2008) 335.

[4] M. Fujiki et al., J Gen Virol 87 (2006) 2699.

[5] T. Meshi et al., Virol 187 (1992) 809.

[6] K. Esau and J. Cronshaw, J Cell Biol 33 (1967) 665.

[7] Thanks to Dr. Jotham Austin, University of Chicago, for electron tomography. 

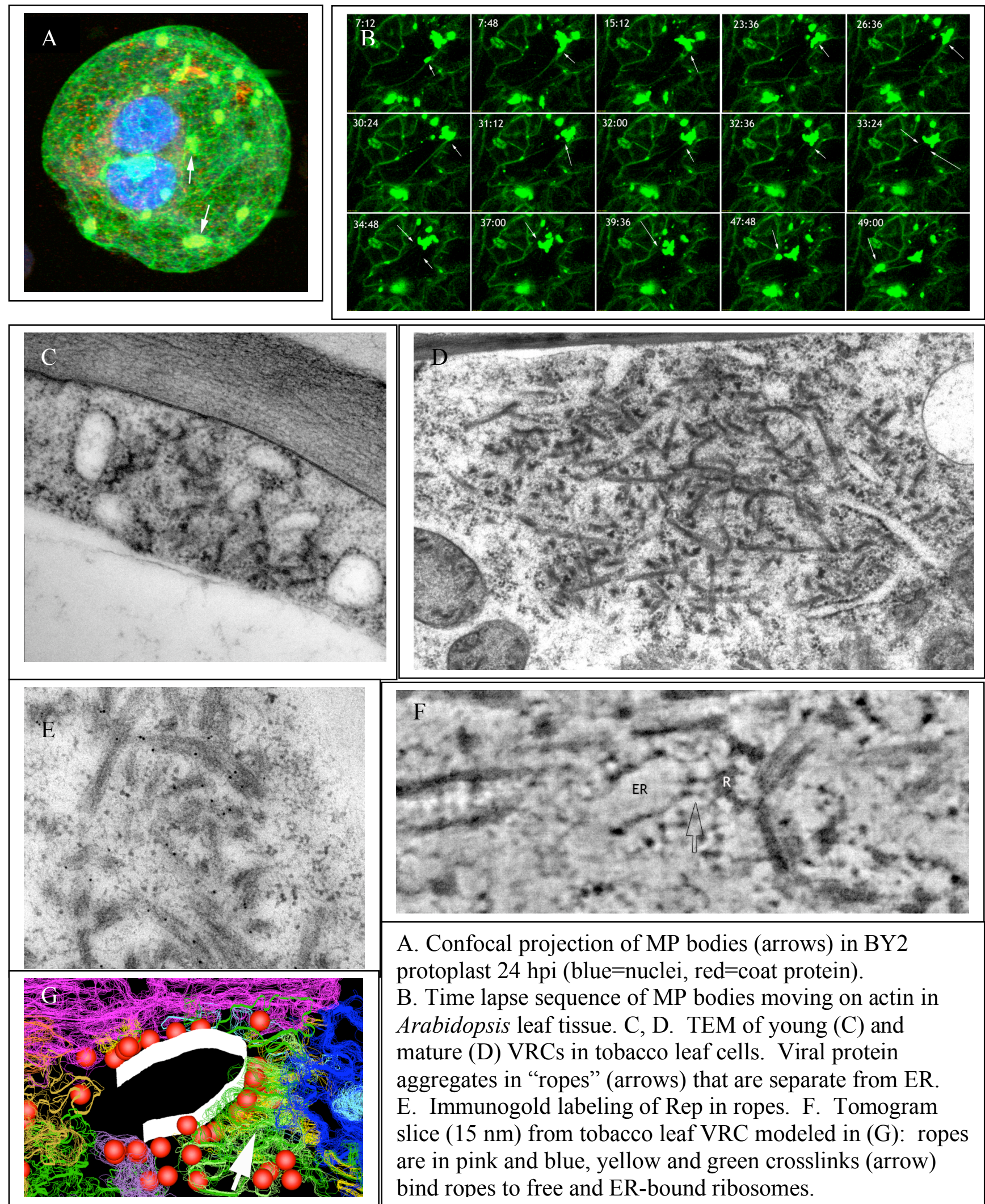

A. Confocal projection of MP bodies (arrows) in BY2 protoplast $24 \mathrm{hpi}$ (blue $=$ nuclei, red $=$ coat protein). B. Time lapse sequence of MP bodies moving on actin in Arabidopsis leaf tissue. C, D. TEM of young (C) and mature (D) VRCs in tobacco leaf cells. Viral protein aggregates in "ropes" (arrows) that are separate from ER. E. Immunogold labeling of Rep in ropes. F. Tomogram slice $(15 \mathrm{~nm})$ from tobacco leaf VRC modeled in $(\mathrm{G})$ : ropes are in pink and blue, yellow and green crosslinks (arrow) bind ropes to free and ER-bound ribosomes. 